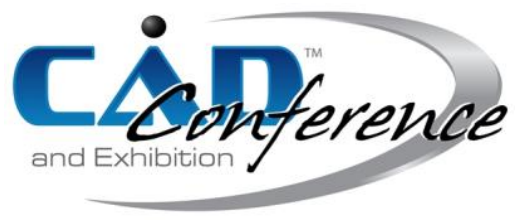

Title:

Rendering DICOM data with B-spline surfaces

Authors:

Les A. Piegl, lespiegl@usf.edu, University of South Florida

William L. Mondy,wmondy@usf.edu, University of South Florida

Keywords:

Medical imaging, image analysis, DICOM data, B-splines, surfaces, bio-engineering

DOI: 10.14733/cadconfP.2021.93-97

Introduction:

Image-based medical diagnosis follows a simple process. First, the data is acquired by a body scanner that measures the linear attenuation of rays passing through the body. The measure that is widely used is the Hounsfield unit (HU) of radiodensity [9]. Typical scanners, such as a CT scanner, can return about 4,000 levels of $\mathrm{HU}$. Once the scanning is done, the raw numbers and other patient-related information are placed in a DICOM (Digital Images and Communications in Medicine) file. The image intensities in the DICOM file are displayed on a computer monitor or on a film for the physician to study. So, the pipeline is simply this: scanner - DICOM - display - eyes. This seems like a reasonable process and is being used routinely all over the world. Unfortunately, they may not show enough information to render a proper diagnosis because the rendered images reveal only a tiny percentage of the DICOM file information.

There have been plenty of papers regarding DICOM data visualization in medicine. This paper does not intend to survey the field; however, a few papers are relevant for this research (an excellent survey on available software packages is found in [5]). The papers can be grouped into three main categories: (1) visualization toolkits [3], [4], [6], (2) 3D reconstruction [1], [2], [7], [11], and (3) multidimensional views with possible overlays [10], [12]. The literature shows that all methods use images either for visualization or 3D reconstruction (voxel and surface-based). The rich content from the DICOM file is lost or compressed to the capabilities of imaging devices.

\title{
The Eyes:
}

Our eyes are wonderful organs that allow us to experience the beauty of the world; however, they have severe limitations. Some of the limitations are welcome, and others are troublesome. One of the welcome limitations is their inability to distinguish points at a distance. It is welcome because it gave us color graphics; we place three dots, red, green, and blue, next to each other, and the eyes, not being able to distinguish them, fuse them into a color sensation. The second welcome limitation is that it cannot distinguish fast-moving images. This gave us motion pictures, where images that are moving faster than about 30 times a second are fused together into moving objects.

On the unwelcome side, we have problems with color. Each of us perceives different color sensations (in technical jargon, each person has a different color gamut), and many of us are just color blind. Then there are the optical illusions; one of the most well-known ones is the Mach band effect, named after physicist Ernst Mach. It is based on the lateral inhibition of the eye; when bright and dark areas meet, the bright appears to be brighter and the dark darker. This happens quite often in medical images where bone structures meet other tissue; the boundaries can appear brighter (and thicker). The third limitation, and most relevant to this paper, is that the eye can only see a certain number of shades in a given color spectrum. A simple test can show that for the eyes to distinguish two gray 
boxes clearly, we may have to increase the intensity by as much as eight levels. This means that out of 256 shades of gray, we can see only about 32! This also means that our eyes are able to see just about $1 \%$ of the over 4,000 levels of intensities that the CT scanner returns.

While our eyes are remarkable instruments, it is reasonable to assume that most people, including radiologists, can only see about 30-40, maybe 50 shades of gray. This also means that the information about the tissues is severely compressed when inspecting medical images. This also means that the perceived images do not necessarily reflect the reality inside the body.

\section{B-spline Fitting to DICOM Data:}

A B-spline surface of degrees $(p, q)$ is defined as follows [8]:

$$
S(u, v)=\sum_{i=0}^{n} \sum_{j=0}^{m} N_{i, p}(u) N_{j, q}(v) P_{i, j}
$$

where $P_{i . j}=\left(x_{i, j}, y_{i, j}, z_{i, j}\right)$ are the control points, and $N_{i, p}(u), N_{j, q}(v)$ are the normalized B-spline basis functions defined over the knot vectors

$$
U=\{\underbrace{u_{0}=\cdots u_{p}}_{p+1}, u_{p+1}, \cdots, u_{n}, \underbrace{u_{r-p}=\cdots=u_{r}}_{p+1}\} \quad V=\{\underbrace{v_{0}=\cdots v_{q}}_{q+1}, v_{q+1}, \cdots, v_{m}, \underbrace{v_{s-q}=\cdots=v_{s}}_{q+1}\}\left(\begin{array}{l}
r=n+p+1 \\
s=m+q+1
\end{array}\right)
$$

B-spline surfaces can be constructed in many ways, and the two most common ones are interpolating or approximating a set of points. In this paper, we used approximation to within tolerance to capture as much detail from the DICOM file as there is to capture. The point set was defined as follows:

$$
Q_{i, j}=\left(x_{i, j}, y_{i, j}, h_{i, j}\right), i=0, \cdots, k ; j=0, \cdots, l
$$

where $\left(x_{i, j}, y_{i, j}\right)$ represent a grid of points on the plane, and $h_{i, j} \approx I_{i, j}$. For each grid point, there is an elevation that is proportional to the intensity found in the DICOM file. To solve the approximation problem, the following details need to be worked out:

- Data smoothing, i.e., eliminating the noise in the DICOM file.

- Segmenting, or decomposing, the rows/columns of data to determine the degrees of freedom (number of control points and knots) needed to achieve the required accuracy.

- $\quad$ Fitting rows (or columns) of data with B-spline curves, followed by fitting the control points of the fitted curves to obtain the surface. This is a lofting-type algorithm that creates a surface from a set of curves.

- Checking for accuracy to make sure that the fitted surface faithfully represents the DICOM data. If the accuracy requirement is not met, the degrees of freedom need to be increased, and the surface needs to be recomputed.

Let us start with data smoothing.

\section{Data Smoothing}

To lessen the noise in the DICOM file, smoothing can be applied. A corner-cutting type algorithm, using repeated subdivision, can be applied in either the $\mathrm{x}$ - or the $\mathrm{y}$-direction or alternated between them. Fitting surfaces with or without smoothing can produce significant variations in accuracy as well as in appearance, especially if the amount of smoothing is too high. So, while smoothing does a great job at eliminating the noise, it also eliminates (smooths out) details from the original data.

\section{Data Decomposition}

The DICOM data file represents a matrix of intensities that we turn into terrain-type data. The fitting operates on rows (or columns) of this data by fitting curves to the data points. While B-spline (leastsquares) approximation is a relatively simple task, approximating within tolerance is still challenging. To obtain the minimum number of control points while maintaining accuracy and proper parametrization requires the following:

- the number of control points and knots; and 
- the positions of the knots as derived from an appropriate parametrization.

So, for each row (or column) of data points, we need to segment or decompose these points into a set of points so that each set represents a simple shape, easily fit by a B-spline segment. In high curvature areas, the algorithm should put smaller segments, whereas low curvature areas can be approximated with longer running curve pieces. Figure 1 illustrates the idea:

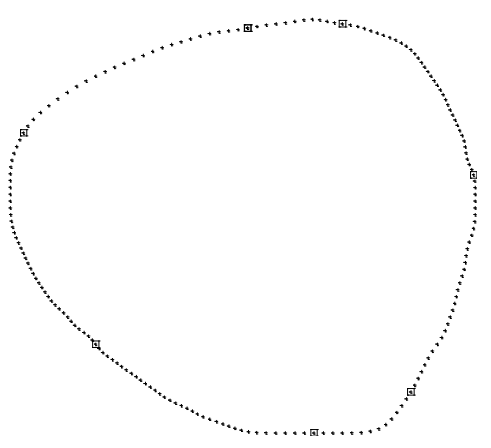

Fig. 1: Bezier decomposition of a data set.

The idea in determining a reasonable segmentation of the data points is to fit a set of Bezier curves to the data so that they approximate the points to within the required tolerance. These curves will not be joined smoothly as they are fitted individually. However, we are not seeking a smooth piecewise Bezier approximation; we are after the degrees of freedom and the locations of the junctions that are inherent in the Bezier decomposition. The algorithm finds the longest-running Bezier segment from a start index to an end index so that the arc approximates the data to within a given tolerance.

\section{B-spline Fitting and Error Control}

Using the Bezier decomposition, we obtained the required degrees of freedom and the location of the knots. The rows of data points are fitted within a tolerance, followed by fitting the fitted curves' control point. The error is controlled by computing the parametric error to avoid high compute time and non-convergence. If the error condition is not satisfied, we return to the decomposition phase and get a new Bezier decomposition to within a tighter tolerance.

Fitting B-spline surfaces to the DICOM data has a few distinct advantages. First, they are not subject to visualization or optical artifacts. They are 3-D surfaces that can be looked at in any position with various lighting conditions. Second, there is no loss of data. The DICOM data is precisely fit, and the resulting surface does pass near/through every data point. Third, if the DICOM file's noise is to be eliminated, smoothing can be applied to any level. In fact, a high level of smoothing can "melt" the surface down to the plane (see the example below). Last but not least, and this is most important, the surfaces are scalable without any loss. Image scaling is done via pixel replication, which produces blurred pictures. That is, zooming into a medical image won't reveal any more details; in fact, it will make the image worst. Zooming into a B-spline surface gives all the details there are in the DICOM file.

\section{Example:}

Let us investigate a cross-section of the knee. Figure 2 shows the knee CT image on the left and the corresponding B-spline surface on the right. The CT image has a 512 × 512 resolution, and the maximum intensity is 2,412. The B-spline surface reveals an incredible amount of detail. One can also observe the Mach band effect where the bone meets the muscle; the bone boundary appears lighter and the muscle darker. On the other hand, the B-spline surface shows that the outer and the inner boundaries (the elevations) are sharp. The image's intensities bleed over to the adjacent parts, whereas the intensities in the surface result in higher elevations, eliminating the optical illusions. 

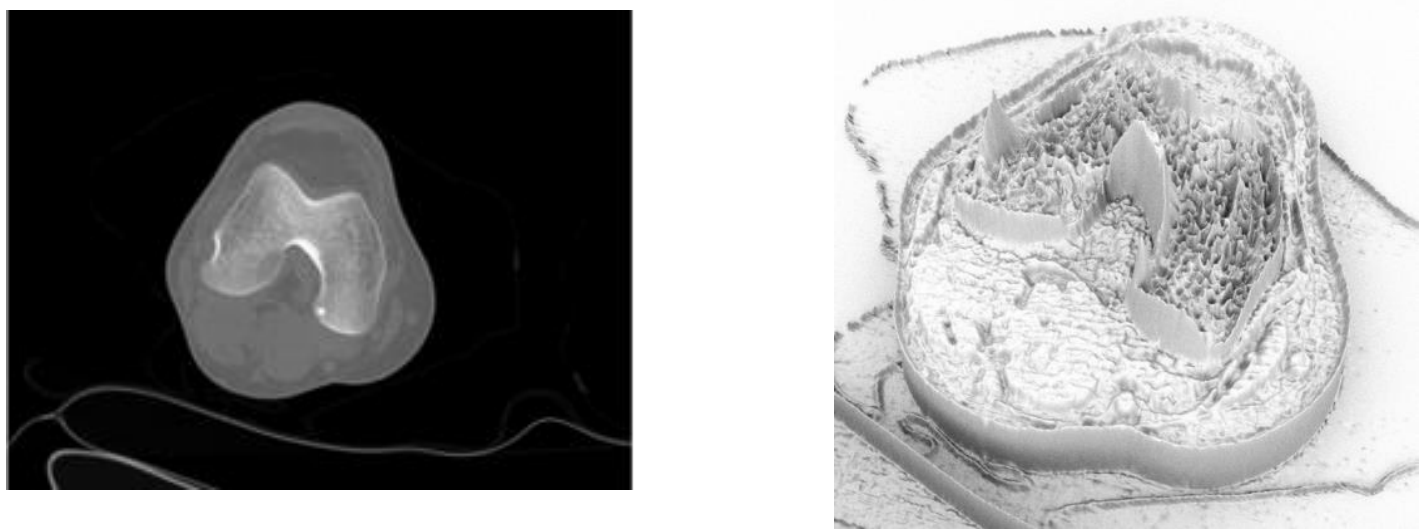

Fig. 2: Knee CT and corresponding B-spline surface.
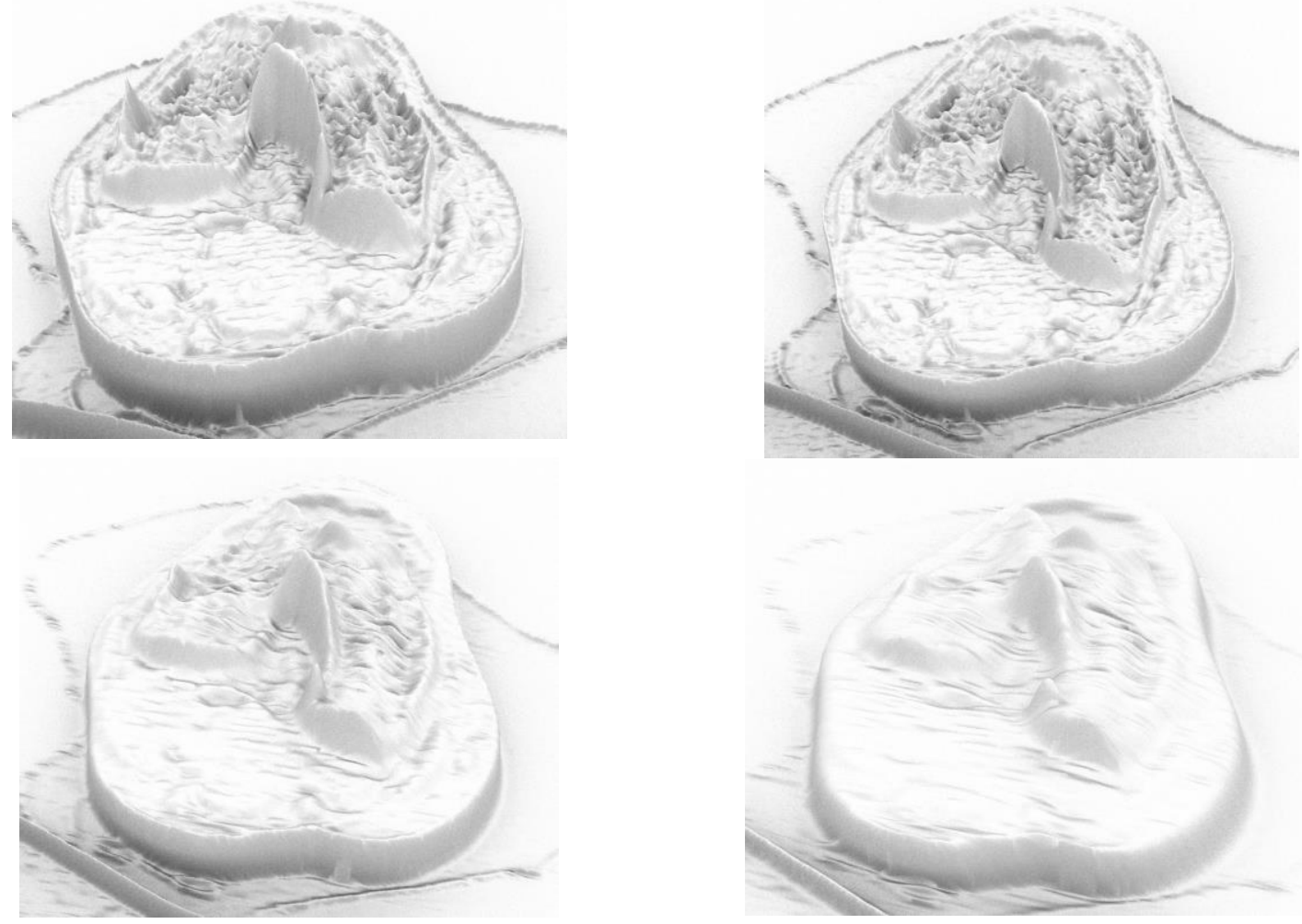

Fig. 3: The effect of denoising: top left, 1; top right, 2; bottom left, 15; bottom right, 50.

Figure 3 illustrates the process of denoising, i.e., eliminating sudden changes in intensities. The top left figure shows one denoising level, the top right has two levels, whereas the bottom left is denoised 15 times, and the bottom right 50 times. It is pretty evident that as the denoising continues, details gradually disappear, and the B-spline surface kind of "melts" away. This is the same phenomenon 
when the 1 to 2,412 intensities are compressed down to 1-256 on the display and finally down to 1-40 in our eyes. Information simply disappears!

From this simple example, it should be clear that denoising is not the way to eliminate significant variations in scanned intensities. Even one-to-two denoising levels reduce details that could be useful in medical diagnosis (compare Figure 2 right to Figure 3 bottom). If image compression causes loss of information and visual artifacts, and if denoising creates a "melting" effect, then so far, the original DICOM data is still the clear winner when analyzing tissues using linear attenuation of radiation.

Conclusions:

In this paper, we introduced B-spline surfaces to illustrate some of the shortcomings of image-based diagnosis using MRI, CT, and X-ray images. The surfaces clearly show that images alone may be inadequate in finding enough details when conditions are not in their advanced stage. The images suffer from visual artifacts, they are non-scalable, and the eyes can only see about $1 \%$ of the available information in the DICOM file. On the other hand, B-spline surfaces can show all the data. The maximum height can be adjusted to show as much detail as needed. The surface is scalable, and features not present in the images reveal themselves at specific heights of the surfaces.

\section{References:}

[1] Chiorean, L-D.; Szasz, T.; Vaida, M-F.; Voina, A.: 3D reconstruction and volume computing in medical imaging, Acta Technica Napocensis, 52(3), 2011, 18-24.

[2] Fisher, F.; Selver, M. A.; Gezer, S.; Dicle, O.; Hillen, W.; Systematic parametrization, storage, and representation of volumetric DICOM data, Journal of Med. Biol. Eng., 35, 2015, 709-723. https://doi.org/10.1007/s40846-015-0097-5

[3] Gambino, O.; Rundo, L.; Cannella, V.; Vitabile, S.; Pirrone, R.: A framework for data-driven adaptive GUI generation based on DICOM, Journal of Biomedical Informatics, 88, 2018, 37-52. https://doi.org/10.1016/j.jbi.2018.10.009

[4] Gutman, D. A.; Dunn, W. D.; Cobb, J.; Stoner, R. M.; Kalpathy-Cramer, J.; Erickson, B.: Web-based tools for visualizing image data and development of XNATView, a zero-footprint image viewer, Frontiers in Neuroinformatics, 8(53), 2014, 1-10. https://doi.org/10.3389/fninf.2014.00053

[5] Haak, D.; Page, C-E.; Desemo, T. M.: A survey of DICOM viewer software to integrate clinical research and medical imaging, Journal of Digital Imaging, 29(2), 2016, 206-215. https://doi.org/10.1007/s10278-015-9833-1

[6] Klotzer, J. D.; Padate, P. B.; Jester, E. T.; Arlagada, V. K. R.: Zero footprint DICOM images viewer, US Patent Pub. No: US 2014/0143298 A1, 2014.

[7] Liu, S.; Liao, W.; Yu, Q.; Cheng, X.; Dai, N.; Zhang, X.: The development of a system for 3D reconstruction from DICOM data and collaborative visualization, Journal of Biomedical Engineering, 24(5), 2007, 1152-1156.

[8] Piegl, L. A.; Tiller, W.: The NURBS Book, Springer-Verlag, New York, NY, 1997. https://doi.org/10.1007/978-3-642-59223-2

[9] Romans, L. E.: Computed Tomography for Technologists: A Comprehensive Text, Wolters Kluwer, Philadelphia, PA, 2019.

[10] Rosset, A.; Spadola, L.; Ratib, O.: OsiriX: an open-source software for navigating in multidimensional DICOM images, Journal of Digital Imaging, 17(3), 2004, 205-216. https://doi.org/10.1007/s10278-004-1014-6

[11] Stutsman, S.; Napoli, J.; Chun, W-S.: 3D volume construction from DICOM data, US Patent Pub. No.: US 2006,0056680 A1, 2006.

[12] Taka, S. J.; Srinivasan, S.: NIRViz: 3D visualization software for multimodality optical imaging using visualization toolkit (VTK) and insight segmentation toolkit (ITK), Journal of Digital Imaging, 24(6), 2011, 1103-1111. https://doi.org/10.1007/s10278-011-9362-5 